\title{
(Anomalous) Gauge boson couplings
}

\section{Evelyne Delmeire ${ }^{* \dagger}$}

\author{
Université de Genève, Switzerland
}

E-mail: Evelyne.Delmeiredcern.ch

\begin{abstract}
During the LEP2 period the $\mathrm{e}^{+} \mathrm{e}^{-}$collider increased its center-of-mass energy from $161 \mathrm{GeV}$ up to $209 \mathrm{GeV}$. A total integrated luminosity of approximately $700 \mathrm{pb}^{-1}$ was recorded per experiment. Massive $\mathrm{W}$ bosons are dominantly produced in pairs via $e^{+} e^{-}$interactions and gauge couplings involving the charged gauge bosons $\mathrm{W}^{+}$and $\mathrm{W}^{-}$, and the neutral gauge bosons $\gamma$ and $\mathrm{Z}$, are studied by the LEP experiments. The LEP measurement of the coupling of the $\mathrm{W}$ boson to the neutral gauge bosons, $g_{1}^{Z}=0.984_{-.019}^{+.022}, \kappa_{\gamma}=0.973_{-.045}^{+.044}$, and $\lambda_{\gamma}=-0.028_{-.021}^{+.020}$, are in agreement with the Standard Model expectation $g_{1}^{Z}=1, \kappa_{\gamma}=1$, and $\lambda_{\gamma}=0$. Couplings between tree and four neutral gauge bosons are forbidden by the Standard Model. No evidence has been found for couplings of three neutral gauge bosons, parametrized by $f_{4,5}^{Z, \gamma}$ and $h_{1,2,3,4}^{Z, \gamma}$. Limits are derived on couplings of four gauge bosons, parametrized by $a_{0}^{Z, W} / \Lambda^{2}, a_{n}^{W} / \Lambda^{2}$ and $a_{c}^{Z, W} / \Lambda^{2}$ where $\Lambda$ represents the energy scale for new physics. A lower limit on the techni- $\rho$ mass of $600 \mathrm{GeV} / \mathrm{c}^{2}$ is set at $95 \%$ confidence level by the ALEPH experiment.
\end{abstract}

International Europhysics Conference on High Energy Physics

July 21st - 27th 2005

Lisboa, Portugal

\footnotetext{
* Speaker.

† On behalf of the LEP Collaborations
} 

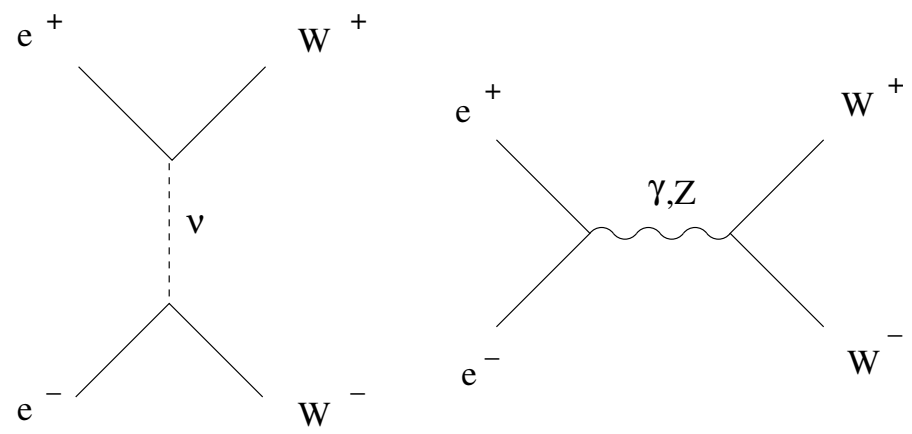

Figure 1: The CCO3 Feynman diagrams for $W$-pair production. Left : t-channel $v$-exchange, Right : s-channel $\gamma / Z$-exchange

At LEP2, the four LEP experiments ALEPH, DELPHI, L3 and OPAL [1] recorded approximately $700 \mathrm{pb}^{-1}$ of total integrated luminosity in the center-of-mass energy range from $161 \mathrm{GeV}$ up to $209 \mathrm{GeV}$. Massive $\mathrm{W}$ bosons are dominantly produced in pairs via $e^{+} e^{-}$interactions and gauge couplings involving the charged gauge bosons $\mathrm{W}^{+}$and $\mathrm{W}^{-}$, and the neutral gauge bosons $\gamma$ and $\mathrm{Z}$, are studied by the LEP experiments.

The non-Abelian $S U(2)_{L} \otimes U(1)_{Y}$ gauge structure of the Standard Model [2] introduces interactions, at tree level, between three or four charged and neutral gauge bosons, called triple and quartic gauge couplings. At LEP, the charged Triple Gauge Couplings (TGC) WWZ and WW $\gamma$ are directly observed. The Quartic Gauge Couplings (QGC) WWWW and WWZZ are not accessible at LEP as the center-of-mass energy is not sufficient, while WWZ $\gamma$ and WW $\gamma \gamma$ are statistically negligible. Neutral triple and quartic gauge couplings between neutral gauge bosons are forbidden by the Standard Model. A coupling is called anomalous if it deviates from its value predicted by the Standard Model or if a new physics process introduces a new vertex at tree level or in the loop corrections that already exist in the Standard Model. The deviations of possible new physcis like supersymmetry, technicolor, composite $W$ bosons, etc, are estimated of the same order of the already existing Standard Model radiative corrections i.e. $10^{-3}$, depending on the coupling parameter.

\section{Charged Triple Gauge Couplings}

The Standard Model predicts the contribution of three charged current Feynman diagrams, referred to as $\mathrm{CC} 03$, and presented in Figure 1. The $t$-channel $v$-exchange diagram (Left), is dominant at the $W W$ threshold. The $s$-channel $\gamma$ and $Z$-exchange diagrams (Right) contain the triple gauge boson vertex $Z W W$ or $\gamma W W$. Figure 2 shows the LEP measurement of the $W$-pair production cross section as function of the center-of-mass energy $\sqrt{s}$. The good agreement between the LEP data and the Standard Model prediction as calculated by the YFSWW [3] and the RACOONWW [4] Monte Carlo's, gives a direct confirmation of the existence of the $\gamma W W$ and the $Z W W$ vertices, predicted by the Standard Model. Also the theoretical prediction for the total $W$ pair production cross section under different hypotheses is shown. The dotted line shows the contribution to the $W$ pair production cross section of the $t$-channel $v$-exchange process only. The $t$-channel $v$-exchange dominates around the $W W$ threshold, but grows rapidly with increasing energy and could eventually 


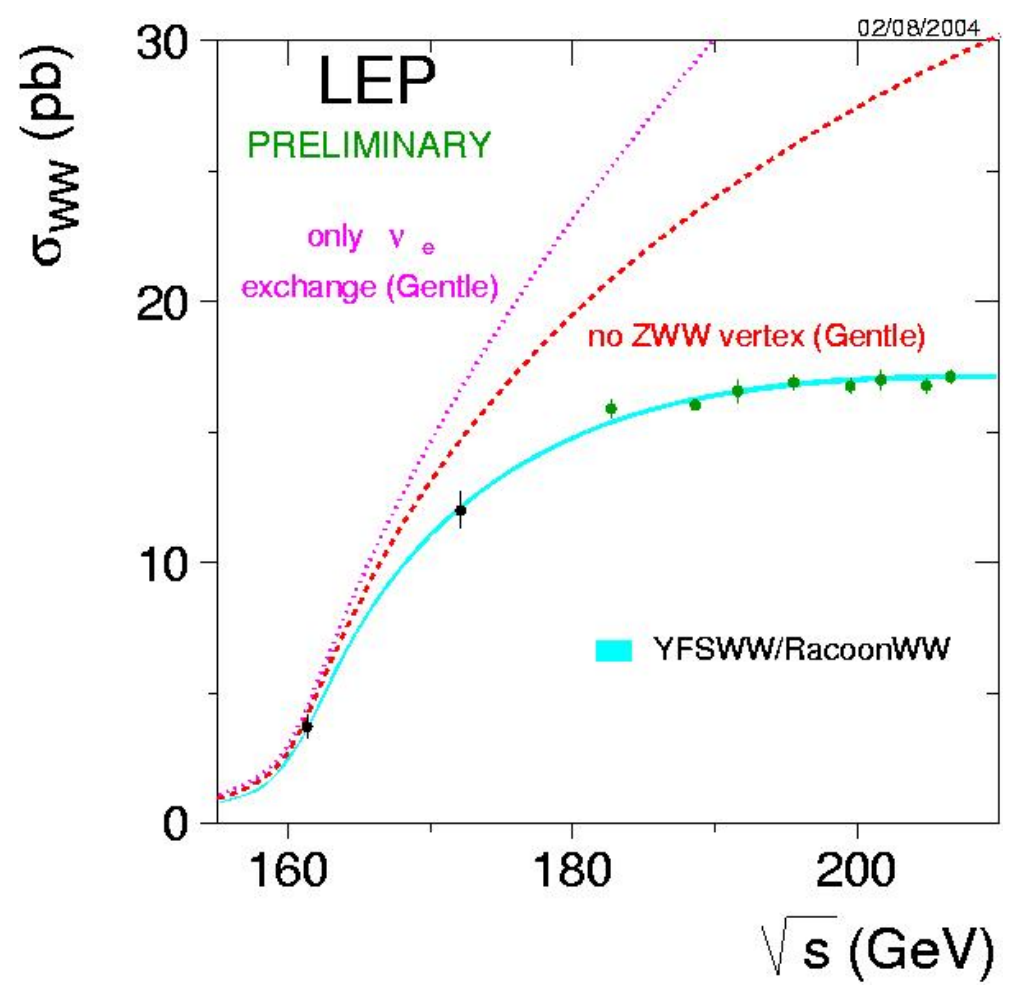

Figure 2: The LEP measurement of the total $W$ pair production cross section as function of the center-ofmass energy $\sqrt{s}$.

violate unitarity at large energy scale. According to the Standard Model the $t$-channel growth is compensated by the contribution of the $s$-channel $\gamma / Z$-exchange with such a coefficient that the cross section decreases with increasing center-of-mass energy. The dashed line represents the behaviour in absence of the $Z W W$ vertex. In this case there would be unitarity violation too. Therefore, the $\gamma W W$ vertex alone is not sufficient to make the cross section finite, but the destructive interference of all three diagrams is needed to describe the LEP data.

The most general Lorentz invariant Lagrangian involving $W W V(V=\gamma, Z)$ vertices can be parametrized by 14 parameters [5]

$$
\begin{gathered}
i \mathscr{L}^{W W V} / g_{W W V}=g_{1}^{V} V^{\mu}\left(W_{\mu v}^{-} W^{+v}-W_{\mu \nu}^{+} W^{-v}\right)+\kappa_{V} W_{\mu}^{+} W_{v}^{-} V^{\mu v} \\
+\frac{\lambda_{V}}{M_{W}^{2}} V^{\mu v} W_{v}^{+\rho} W_{\rho \mu}^{-}+i g_{5}^{V} \varepsilon_{\mu v \rho \sigma}\left[\left(\partial^{\rho} W^{-\mu}\right) W^{+v}-W^{-\mu}\left(\partial^{\rho} W^{+v}\right)\right] V^{\sigma} \\
+i g_{4}^{V} W_{\mu}^{-} W_{v}^{+}\left(\partial^{\mu} V^{v}-\partial^{v} V^{\mu}\right)-\frac{\tilde{\kappa}_{V}}{2} W_{\mu}^{-} W_{v}^{+} \varepsilon^{\mu v \rho \sigma} V_{\rho \sigma}-\frac{\tilde{\lambda}_{V}}{2 M_{V}^{2}} \mathrm{~W}_{\rho \mu}^{-} \mathrm{W}_{v}^{+\mu} \varepsilon^{v \rho \alpha \beta} \mathrm{V}_{\alpha \beta}
\end{gathered}
$$

where $g_{1}^{V}, \kappa_{V}, \lambda_{V}$ and $g_{5}^{V}$ are CP-conserving couplings while $g_{4}^{V}, \tilde{\kappa}_{V}$ and $\tilde{\lambda}_{V}$ are CP-violating. Assuming $\mathrm{CP}$-conservation and electromagnetic gauge invariance of the Lagrangian, five parameters are left $: g_{1}^{Z}, \kappa_{Z}, \kappa_{\gamma}, \lambda_{Z}$ and $\lambda_{\gamma}$. The custodial $S U(2)$ symmetry of the Lagrangian imposes the constraints

$$
\kappa_{Z}=g_{1}^{Z}-\left(\kappa_{\gamma}-1\right) \tan ^{2} \theta_{W} \quad \lambda_{\gamma}=\lambda_{Z}
$$



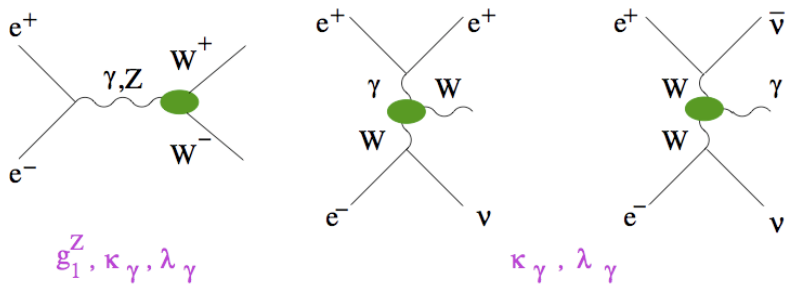

Figure 3: The processes sensitive to $Z W W$ and $\gamma W W$ triple gauge couplings : $\mathrm{W}$-pair, single $\mathrm{W}$ and single photon production.

where $\theta_{W}$ is the weak mixing angle. Three free parameters are left $: g_{1}^{Z}, \kappa_{\gamma}$ and $\lambda_{\gamma}$. They are related to the magnetic dipole and electric quadrupole moment of the W. The Standard Model predicts their values to be $g_{1}^{Z}=\kappa_{\gamma}=1$ and $\lambda_{\gamma}=0$ at tree level.

The triple gauge couplings are studied in $\mathrm{W}$-pair production, sensitive to all three parameters, and single $\mathrm{W}$ and single photon production which are sensitive to $\kappa_{\gamma}$ and $\lambda_{\gamma}$ only. The corresponding Feynman diagrams are presented in Figure 3. In general W-pair production is the most sensitive process, except for the parameter $\kappa_{\gamma}$, where single $\mathrm{W}$ poduction has the maximum sensitivity. The single $\mathrm{W}$ and single $\gamma$ process are included in the TGC analysis by some LEP experiments.

A deviation from a coupling value predicted by the Standard Model would modify the total cross section, the shape of the $\mathrm{W}$ production angle $\theta_{W}$, the polar angle $\theta_{f}^{\star}$ and azimuthal angle, $\phi_{f}^{\star}$ of the W-decay fermion in the corresponding $\mathrm{W}$ restframe. These angles are presented in Figure 4 together with the distribution of the W production angle as measured by the ALEPH experiment in fully hadronic $\mathrm{W}$-pair events. The expected distribution in presence of an anomalous coupling $\lambda_{\gamma}= \pm 0.2$ is also indicated.

The couplings are extracted by a maximum likelihood fit to the angular distributions (DELPHI, L3) or by a $\chi^{2}$-fit to Optimal Observables distributions (ALEPH, OPAL). The results from each LEP experiment are then combined using a log-likelihood method [6]. A one-parameter fit [1] is performed for the PDG 2005 including published results from ALEPH, L3 and OPAL and is presented in Figure 5.

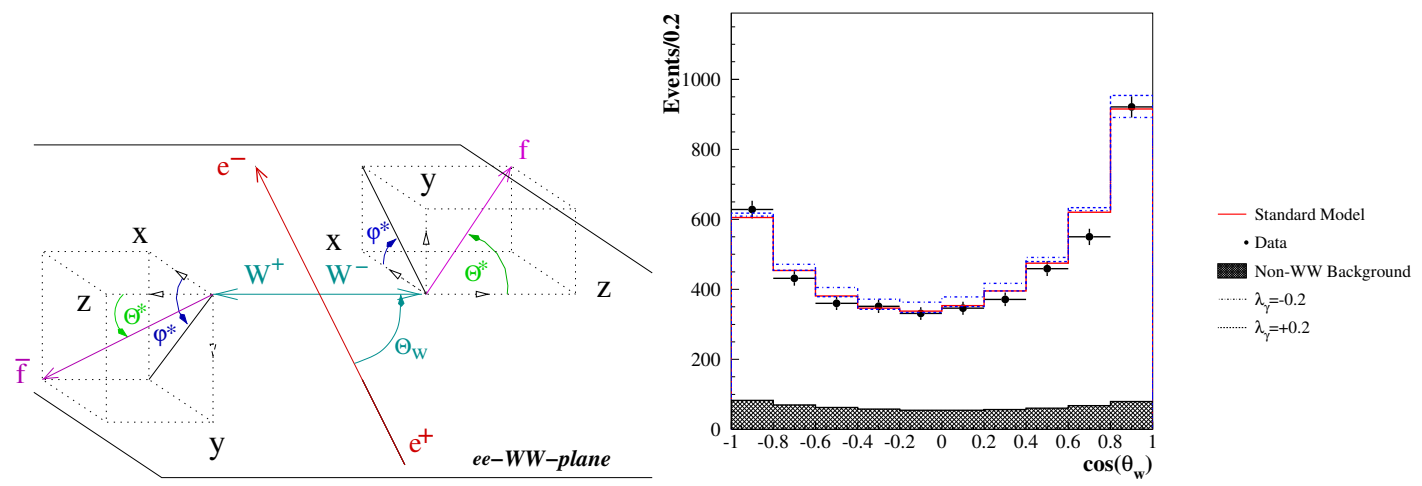

Figure 4: Left : The definition of the angles used in the coupling extraction. Right: The $\mathrm{W}$ boson production angle measured by the ALEPH experiment in fully hadronic $\mathrm{W}$-pair events. 

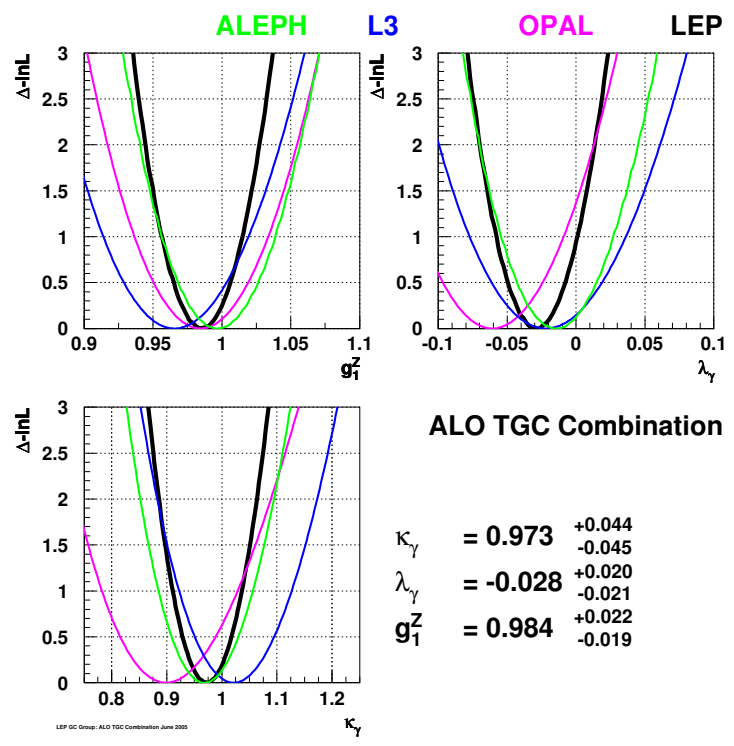

ALO TGC Combination

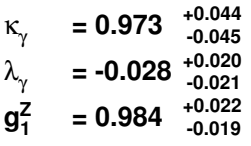

Figure 5: The -log $\mathscr{L}$ curves of the one-parameter fit for the PDG 2005 including published results from ALEPH, L3 and OPAL. Each coupling parameter is varied individually fixing the other two parameters to their Standard Model expectation.

\begin{tabular}{|c||c|c|c|}
\hline Source & $g_{1}^{Z}$ & $\lambda_{\gamma}$ & $\kappa_{\gamma}$ \\
\hline $\mathscr{O}(\alpha)$ corrections & 0.010 & 0.010 & 0.020 \\
Hadronisation & 0.004 & 0.002 & 0.004 \\
Bose-Einstein correlation & 0.005 & 0.004 & 0.009 \\
Colour Reconnection & 0.005 & 0.004 & 0.010 \\
$\sigma_{W W}$ prediction & 0.003 & 0.005 & 0.014 \\
$\sigma_{\text {single } W}$ prediction & & & 0.011 \\
\hline
\end{tabular}

Table 1: The sources of experiment correlated systematic uncertainties in the LEP combination and their effect on the combined fit results.

The fit results

$$
g_{1}^{Z}=0.984_{-.019}^{+.022} \quad \kappa_{\gamma}=0.973_{-.045}^{+.044} \quad \lambda_{\gamma}=-0.028_{-.021}^{+.020} .
$$

are in agreement with the Standard Model prediction. A final LEP combination is forseen once the DELPHI TGC results published. The quoted errors include both statistical and systematic uncertainties. The uncertainty is dominated by the statistical component. The largest contribution to the systematic uncertainty comes from the $\mathscr{O}(\alpha)$ radiative corrections. The experiment correlated systematic uncertainties in the combined fit results are presented in Table 1. 
The ALEPH experiment also performs unconstrained fits assuming no relation between the 28 TGC parameters. No deviation from the Standard Model prediction is observed [7].

The L3 experiment uses the TGC measurement to study spacial extention of the $W$ boson, approximated by an ellipsoid with longitudinal radius $a$ and transverse radius $b$. The size and radius would be related to the TGC by $R_{W} \equiv(a+b) / 2=\left(\kappa_{\gamma}+\lambda_{\gamma}-1\right) / m_{W}[8]$ and $\Delta_{W} \equiv\left(a^{2}-b^{2}\right) / 2=$ $(5 / 4)\left(\kappa_{\gamma}-\lambda_{\gamma}-1\right) / m_{W}^{2}$ [9] where $m_{W}$ is the $W$-boson mass. There is no evidence that the $W$ boson would be an extended object [10]

$$
\begin{gathered}
R_{W}=(0.3 \pm 1.9) \times 10^{-19} \mathrm{~m} \\
\Delta_{W}=(0.89 \pm 0.83) \times 10^{-36} \mathrm{~m}^{2}
\end{gathered}
$$

\subsection{Measurement of the W boson Spin Density Matrix}

The Spin Density Matrix (SDM) method $[11,12]$ is used to set direct limits on CP-violating couplings, absent in the Standard Model.

Considering the helicity, the $\mathrm{W}$-pair production process is written as

$$
\mathrm{e}^{+}\left(\lambda^{\prime}\right) \mathrm{e}^{-}(\lambda) \rightarrow \mathrm{W}^{+}\left(\tau_{2}\right) \mathrm{W}^{-}\left(\tau_{1}\right)
$$

where $\lambda, \lambda^{\prime}$ are the helicity of the electron (positron). In the high energy limit, where we can neglect the electron mass, the helicity of the positron is opposite to the electron's helicity: $\lambda^{\prime}=-\lambda$. The helicities of the $\mathrm{W}^{-}$and the $\mathrm{W}^{+}$, denoted by $\tau_{1}$ and $\tau_{2}$ respectively, take the value $\tau= \pm 1$ for transversely polarised $\mathrm{W}$ bosons and the value $\tau=0$ for $W$ bosons with a longitudinal polarisation.

The two-particle joint SDM elements are then defined as [13]

$$
\rho_{\tau_{1} \tau_{1}^{\prime} \tau_{2} \tau_{2}^{\prime}}\left(s, \cos \theta_{\mathrm{W}}\right) \equiv \frac{\sum_{\lambda} F_{\tau_{1} \tau_{2}}^{\lambda}\left(F_{\tau_{1}^{\prime} \tau_{2}^{\prime}}^{\lambda}\right)^{\star}}{\sum_{\lambda, \tau_{1}, \tau_{2}}\left|F_{\tau_{1} \tau_{2}}^{\lambda}\right|^{2}},
$$

where $s$ is the center-of-mass energy and $F_{\tau_{1}}^{\lambda} \tau_{2}$ is the helicity amplitude for the production of a $\mathrm{W}$-pair with helicities $\tau_{1}$ and $\tau_{2}$. The single W SDM elements are obtained by summation over all possible helicities of one of the W's, by convention the $\mathrm{W}^{+}$,

$$
\rho_{\tau_{1} \tau_{1^{\prime}}}^{\mathrm{W}^{-}}\left(s, \cos \theta_{\mathrm{W}^{-}}\right) \equiv \sum_{\tau_{2}} \rho_{\tau_{1} \tau_{1}^{\prime} \tau_{2} \tau_{2}}\left(s, \cos \theta_{\mathrm{W}^{-}}\right)
$$

The SDM elements are constrained by Hermiticity $\rho_{\tau \tau^{\prime}}^{\mathrm{W}^{-}}=\left(\rho_{\tau^{\prime} \tau}^{\mathrm{W}}\right)^{\star}$ and are normalised to unity $\sum_{\tau} \rho_{\tau \tau}^{\mathrm{W}^{-}}=1$. The diagonal elements of the SDM are real and express the probability to produce a $\mathrm{W}^{-}$with a transverse polarisation $\left(\rho_{++}\right.$and $\left.\rho_{--}\right)$or with a longitudinal polarisation $\left(\rho_{00}\right)$. The off-diagonal elements measure the interference between different $\mathrm{W}$ helicity amplitudes. They differ from zero if the $\mathrm{W}$ boson is be produced in a linear superposition of helicity states, predicted by the Standard Model, but would be zero for a W boson produced in a definite helicity state. The off-diagonal elements are complex and provide a test of $\mathrm{CP}$ violation. 

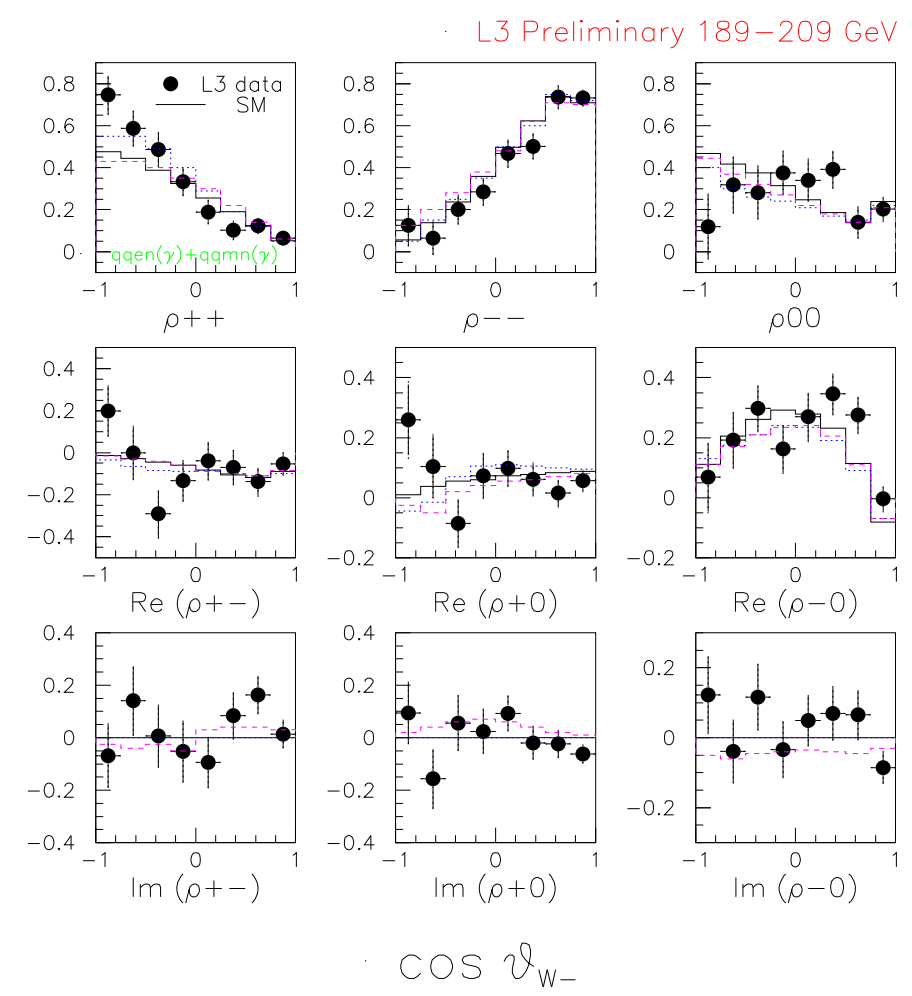

Figure 6: The nine single SDM elements, $\rho_{\tau \tau^{\prime}}^{\mathrm{W}^{-}}$, as a function of $\cos \theta_{\mathrm{W}^{-}}$. The errors bars include statistical and systematic uncertainty.

The SDM elements are calculated in bins of $\cos \theta_{\mathrm{W}^{-}}$using a projection operator method assuming a V-A decay of the $\mathrm{W}$ boson into fermions

$$
\rho_{\tau \tau^{\prime}}^{\mathrm{W}^{-}}(k)=\frac{1}{N_{k}} \sum_{i=1}^{N_{k}} \Lambda_{\tau \tau^{\prime}}^{\mathrm{W}^{-}}\left(\theta_{f}^{\star}, \phi_{f}^{\star}\right)_{i}
$$

where $N_{k}$ is the number of events in the $k$-th bin and where the projection operator $\Lambda_{\tau \tau^{\prime}}^{\mathrm{W}^{-}}$is applied event by event. The reconstructed SDM elements need to be corrected for detector acceptance, resolution effects and background contamination for a direct comparison with the theoretical expectation.

The single W SDM elements measured with the L3 combined $q \bar{q} e v$ and the $q \bar{q} \mu \nu$ data selected at the center-of-mass energies $\sqrt{s}=189-209 \mathrm{GeV}$ are presented in Figure 6 [14]. The measurements for the leptonically decaying $\mathrm{W}^{+}$and $\mathrm{W}^{-}$are combined assuming CPT-invariance. Agreement is found with the Standard Model prediction represented by the solid line. The expected distributions in presence of an anomalous CP-conserving coupling $\kappa_{\gamma}=+1.5$ (blue dotted line) and the CP-violating coupling $\tilde{\lambda}_{Z}=-0.5$ (pink dashed line) are also shown. 


\begin{tabular}{|c||c|c|}
\hline Parameter & $\begin{array}{c}\text { DELPHI } \\
189-208 \mathrm{GeV}\end{array}$ & $\begin{array}{c}\text { OPAL } \\
189 \mathrm{GeV}\end{array}$ \\
\hline$g_{4}^{Z}$ & $-0.30 \pm 0.17$ & $+0.01_{-.33}^{+.32}$ \\
$\tilde{\lambda}_{Z}$ & $-0.08 \pm 0.07$ & $-0.18_{-.16}^{+.24}$ \\
$\tilde{\kappa}_{Z}$ & $-0.03_{-.05}^{+.06}$ & $-0.20_{-.07}^{+.10}$ \\
\hline
\end{tabular}

Table 2: The one-parameter fit results on the CP-violating TGC $g_{4}^{Z}, \tilde{\lambda}_{Z}$ and $\tilde{\kappa}_{Z}$. The error combine the statistical and the systematic uncertainty except for DELPHI where only the statistical component is included.

\begin{tabular}{|c||c|c|c|c|}
\hline Parameter & ALEPH & DELPHI & L3 & OPAL \\
\hline$h_{1}^{\gamma}$ & {$[-.14 ;+.14]$} & {$[-.14 ;+.14]$} & {$[-.06 ;+.06]$} & {$[-.13 ;+.13]$} \\
$h_{2}^{\gamma}$ & {$[-.07 ;+.07]$} & {$[-.09 ;+.09]$} & {$[-.053 ;+.024]$} & {$[-.089 ;+.089]$} \\
$h_{3}^{\gamma}$ & {$[-.069 ;+.037]$} & {$[-.049 ;+.044]$} & {$[-.062 ;+.014]$} & {$[-.16 ;+.00]$} \\
$h_{4}^{\gamma}$ & {$[-.020 ;+.045]$} & {$[-.032 ;+.030]$} & {$[-.004 ; .+045]$} & {$[-.01 ;+.13]$} \\
\hline$h_{1}^{Z}$ & {$[-.23 ;+.23]$} & {$[-.23 ;+.23]$} & {$[-.17 ;+.16]$} & {$[-.22 ;+.22]$} \\
$h_{2}^{Z}$ & {$[-.12 ;+.12]$} & {$[-.14 ;+.14]$} & {$[-.10 ;+.09]$} & {$[-.15 ;+.15]$} \\
$h_{3}^{Z}$ & {$[-.28 ;+.19]$} & {$[-.30 ;+.16]$} & {$[-.23 ;+.11]$} & {$[-.29 ;+.14]$} \\
$h_{4}^{Z}$ & {$[-.10 ;+.15]$} & {$[-.12 ;+.18]$} & {$[-.08 ;+.16]$} & {$[-.09 ;+.19]$} \\
\hline \hline$f_{4}^{\gamma}$ & {$[-.26 ;+.26]$} & {$[-.23 ;+.25]$} & {$[-.28 ;+.28]$} & {$[-.32 ;+.33]$} \\
$f_{5}^{\gamma}$ & {$[-.54 ;+.56]$} & {$[-.52 ;+.48]$} & {$[-.39 ;+.47]$} & {$[-.71 ;+.59]$} \\
\hline$f_{4}^{Z}$ & {$[-.44 ;+.43]$} & {$[-.40 ;+.42]$} & {$[-.48 ;+.46]$} & {$[-.45 ;+.58]$} \\
$f_{5}^{Z}$ & {$[-.73 ;+.83]$} & {$[-.62 ;+.38]$} & {$[-.35 ;+1.03]$} & {$[-.94 ;+.25]$} \\
\hline
\end{tabular}

Table 3: The 95\% confidence level one-dimensional limits set by the LEP experiments on the neutral triple gauge couplings.

The imaginary parts of the off-diagonal elements are not sensitive to CP-conserving couplings and only contribute in presence of tree level CP-violation. This makes the SDM method particularly suitable to measure CP-violating couplings which are extracted by a $\chi^{2}$-fit to the nine SDM-element distributions. The results of the DELPHI [15] and OPAL [16] experiment using semi-leptonic Wpair events are presented in Table 2. No deviation from the Standard Model prediction is observed.

\section{Neutral Triple Gauge Couplings}

Neutral triple gauge couplings do not exist in the Standard Model. The most general Lorentz invariant Lagrangian [5, 17] for the $V V Z(V=\gamma, Z)$ vertex is described by 12 parameters. The couplings $h_{1}^{V}, h_{2}^{V}, h_{3}^{V}$ and $h_{4}^{V}$ are studied at LEP in the $\mathrm{e}^{+} \mathrm{e}^{-} \rightarrow \mathrm{Z} \gamma$ production, while $f_{4}^{V}$ and $f_{5}^{V}$ are accessible in $\mathrm{e}^{+} \mathrm{e}^{-} \rightarrow Z Z$ production. Electromagnetic gauge invariance and Bose symmetry for final states with identical bosons are imposed. The couplings are determined from the angular distributions of the decay products and the total cross section. The one-dimensional limits at $95 \%$ confidence level are $[1,25]$ summarized in Table 3. Both statistical and systematic uncertainties are included. No evidence for anomalous $h$ - and $f$-couplings has been found. 


\begin{tabular}{|c||c|c|c|c|}
\hline Parameter $\left(\mathrm{GeV}^{-2}\right)$ & ALEPH & DELPHI & L3 & OPAL \\
\hline$a_{0}^{W} / \Lambda^{2}$ & {$[-.060 ;+.055]$} & {$[-.020 ;+.020]$} & {$[-.015 ;+.015]$} & {$[-.020 ;+.020]$} \\
$a_{c}^{W} / \Lambda^{2}$ & {$[-.099 ;+.093]$} & {$[-.063 ;+.032]$} & {$[-.048 ;+.026]$} & {$[-.052 ;+.037]$} \\
$a_{n}^{W} / \Lambda^{2}$ & & {$[-.180 ;+.140]$} & {$[-.140 ;+.130]$} & {$[-.160 ;+.150]$} \\
\hline$a_{0}^{Z} / \Lambda^{2}$ & {$[-.012 ;+.019]$} & & {$[-.014 ;+.027]$} & {$[-.007 ;+.023]$} \\
$a_{c}^{Z} / \Lambda^{2}$ & {$[-.041 ;+.044]$} & & {$[-.037 ;+.054]$} & {$[-.029 ;+.029]$} \\
\hline
\end{tabular}

Table 4: The 95\% confidence level one-dimensional limits set by the LEP experiments on the quartic gauge couplings.

\section{Neutral and Charged Quartic Gauge Couplings}

The contribution of the Standard Model quartic gauge couplings is below the LEP sensitivity and therefore, anomalous quartic gauge couplings resulting from new physics beyond the LEP energy are searched for. Deviations are introduced into the Lagrangian $[18,19]$ as effective couplings at a new physics scale $\Lambda$.

Starting from electromagnetic gauge invariance and custodial $S U(2)$ symmetry, the most general Lorentz invariant Lagrangian has 5 parameters. The charged quartic gauge couplings $a_{0}^{W} / \Lambda^{2}$, $a_{n}^{W} / \Lambda^{2}$ and $a_{c}^{W} / \Lambda^{2}$ are studied at LEP in the $\mathrm{e}^{+} \mathrm{e}^{-} \rightarrow \mathrm{W}^{+} \mathrm{W}^{-} \gamma$ reaction, while $a_{0}^{W} / \Lambda^{2}$ and $a_{c}^{W} / \Lambda^{2}$ are also accessible in the $\mathrm{e}^{+} \mathrm{e}^{-} \rightarrow v \bar{v} \gamma \gamma$ process. The neutral quartic gauge couplings $a_{0}^{Z} / \Lambda^{2}$ and $a_{c}^{Z} / \Lambda^{2}$, forbidden by the Standard Model, are searched for in the $\mathrm{e}^{+} \mathrm{e}^{-} \rightarrow \mathrm{Z} \gamma \gamma \rightarrow v \bar{v} \gamma \gamma, \mathrm{q} \overline{\mathrm{q}} \gamma \gamma$ process. Quartic gauge couplings are mainly determined from the photon energy spectrum and the total cross section.

The one-dimensional limits at $95 \%$ confidence level on the charged and neutral quartic gauge couplings set by ALEPH [20], DELPHI [21], L3 [22, 23] and OPAL [24] are summarized in Table 4 . Both statistical and systematic uncertainties are included. No evidence for anomalous quartic gauge couplings has been found.

\section{Techni- $\rho$ Resonance}

The existence of a techni- $\rho$ resonance in $W_{L}^{+} W_{L}^{-}$production, in analogy with the $\rho$-resonance formation in the $e^{+} e^{-} \rightarrow \pi^{+} \pi^{-}$reaction, is probed by the ALEPH experiment through the study of the complex technipion form factor $F_{T}$ [26]

$$
F_{T}=\frac{M_{\rho}^{2}-i \Gamma_{\rho} M_{\rho}}{M_{\rho}^{2}-s-i \Gamma_{\rho} M_{\rho}}
$$

where $M_{\rho}$ and $\Gamma_{\rho}$ are the mass and the width of the techni- $\rho$ respectively. No deviation from the Standard Model prediction is observed. 
The $95 \%$ confidence level intervals on the techni- $\rho$ formfactor are

$$
\begin{gathered}
0.868<\operatorname{Re}\left(F_{T}\right)<1.061 \\
-0.332<\operatorname{Im}\left(F_{T}\right)<0.044
\end{gathered}
$$

which leads to the $95 \%$ confidence level limit $M_{\rho}<600 \mathrm{GeV} / \mathrm{c}^{2}$, assuming the techni- $\rho$ width to be smaller than its mass [7].

\section{Acknowledgments}

I wish to express my gratitude to my collegues from LEP and convenors of the LEP gauge coupling working groups for their support and discussions.

\section{References}

[1] The LEP Collaborations ALEPH, DELPHI, L3, OPAL and the LEP TGC Working Group, LEPEWWG/TGC/2003-01(2003), LEPEWWG/TGC/2005-01(2005), http://lepewwg.web.cern.ch/LEPEWWG/lepww/tgc.

[2] S.L.Glashow, Nucl. Phys. 22 (1961) 579 ;

S.Weinberg, Phys. Rev. Lett. 19 (1967) 1264 ;

A.Salam, in Elementary Particle Theory, ed. N. Svartholm, Stockholm, Almquist and Wiksell (1968), 367.

[3] S.Jadach et al., Precision Predictions for (Un)Stable $W^{+} W^{-}$Pair Production At and Beyond LEP2 Energies, Phys. Rev. D 65 (2002) 093010.

[4] A.Denner et al., Electroweak Radiative Corrections to $e^{+} e^{-} \rightarrow W^{+} W^{-} \rightarrow 4$ fermions in Double Pole Approximation: the RACOONWW Approach, Nucl. Phys. B 587 (2000) 67.

[5] K.Hagiwara, R.D.Peccei, D.Zeppenfeld and K.Hikasa, Nucl. Phys. B 282 (1987) 253.

[6] J.Alcaraz, L3 Note 2718 (2001).

[7] ALEPH Collaboration, Phys. Lett.B 614 (2005) 7.

[8] S.J.Brodsky and S.D.Drell, Phys.Rev. D 22 (1980) 2236.

[9] H.Frauenfelder and E.M.Henley, "Subatomic physics", Englewood Cliffs, New Jersey (1974).

[10] L3 Collaboration, "Measurement of Triple-Gauge-Boson Couplings of the W boson at LEP", Phys. Lett. B 586 (2004) 151.

[11] G.Gournaris, D.Schildknecht and F.M.Renard, Phys. Lett. B 263 (1991) 291.

[12] G.Gournaris, J.Layssac, G.Moultaka and F.M.Renard, Int. J. Mod. Phys. A 8 (1993) 3285.

[13] M.Bilenky, J.L. Knuer, F.M. Renard and D. Schildknecht, Nucl. Phys. B 409 (1993) 22 ; B 419 (1994) 240.

[14] L3 Collaboration, L3 Note 2831, submitted to HEP-EPS2005 (Lisbon).

[15] DELPHI Collaboration, DELPHI 2004-018 CONF 693 (2004), submitted to ICHEP2004 (Beijing).

[16] OPAL Collaboration, G.Abbiendi et al. Eur. Phys. J. C 19 (2001) 1-14.

[17] G.J.Gounaris et al. Phys. Rev. D 61075020 (2000). 
[18] G.Bélanger and F.Boudjema, Phys. Lett. B 288 (1992) 210.

[19] W.J.Stirling and Ghadir Abu Leil, J. Phys. G 21 (1995) 517.

[20] ALEPH Collaboration, "Anomalous Quartic Gauge Couplings in $e^{+} e^{-}$Interactions from 183-209 GeV", submitted to HEP-EPS2003 (Aachen) and ICHEP2004 (Beijing).

[21] DELPHI Collaboration, Eur. Phys. J. C 31 (2003) 139-147.

[22] L3 Collaboration, Phys. Lett. B 527/1-2 (2002) 29-38.

[23] L3 Collaboration, Phys. Lett. B 540/1-2 (2002) 43-51.

[24] OPAL Collaboration, Phys. Rev. D 70 (2004) 032005.

[25] DELPHI Collaboration, 2005-006 CONF 726 (2005), submitted to HEP-EPS2005 (Lisbon) and LP2005 (Uppsala).

[26] T.L.Barklow et al., "Strong coupling electroweak symmetry breaking" in Proceedings of DPF/DPB "Summer Study on New Directions for High Energy Physics (Snowmass 1996)", Snowmass, Colorado, eConf C960625 (1996) STC118. 